\title{
Clinically Amyopathic Dermatomyositis Associated with a Metastatic Undifferentiated Carcinoma in an Adult Filipino Male: A Case Report
}

\author{
Felix Paolo J. Lizarondo ${ }^{1}$ and Eileen Liesl A. Cubillan² \\ ${ }^{1}$ Section of Dermatology, Department of Medicine, Philippine General Hospital, University of the Philippines Manila \\ ${ }^{2}$ Section of Dermatology, Department of Medicine, College of Medicine and Philippine General Hospital, University of the Philippines Manila
}

\begin{abstract}
A 40-year old Filipino male was managed as a case of clinically amyopathic dermatomyositis (CADM) after presenting only with pruritic photodistributed symmetric erythematous-violaceous patches, nail fold telangiectasia and the pathognomonic Gottron papules. There was no clinical evidence of muscle involvement. The patient shortly presented with a metastatic undifferentiated carcinoma on the cervical lymph nodes. This case demonstrates that dermatomyositis can present uncommonly as CADM with associated metastatic undifferentiated carcinoma.
\end{abstract}

Key Words: dermatomyositis, amyopathic, carcinoma, malignancy

E-poster presented at the $27^{\text {th }}$ European Academy of Dermatology and Venereology Congress, September 12-16, 2018, Paris, France.

E-poster presented at the, $41^{\text {st }}$ Annual Convention of the Philippine Dermatological Society, November 7-9, 2018, EDSA Shangri-La, Mandaluyong City, Philippines.

Oral Presentation, Gross and Microscopic Dermatology Symposium, 2019 American Academy of Dermatology Annual Meeting, March 1-5, 2019, Washington DC, USA.

Corresponding author: Felix Paolo J. Lizarondo, MD Section of Dermatology

Department of Medicine

Philippine General Hospital

University of the Philippines Manila

Taft Avenue, Manila 1000, Philippines

Telephone: +632 5548400 local 5105/5106

Email: fjlizarondo@up.edu.ph

\section{INTRODUCTION}

Dermatomyositis (DM) is an autoimmune disease belonging to the spectrum of idiopathic inflammatory myopathies which also includes inclusion body myositis and polymyositis. ${ }^{1}$ It is a rare disease with a worldwide occurrence, with an incidence ranging from 2 to 9 per million across various populations and affects women more than men approximately two to three times more often. ${ }^{1}$

$\mathrm{DM}$ can further be classified into classic DM and clinically amyopathic dermatomyositis (CADM). Patients present with a photodistributed symmetric pruritic confluent violaceous erythematous macules and patches. When specific areas are affected, the hallmark cutaneous findings can be seen such as the shawl sign involving the arms, deltoid, posterior shoulders and neck; V-sign involving the anterior neck and upper chest; and the holster sign involving the lateral aspects of hips and thighs. ${ }^{2}$ The pathognomonic lesions are Gottron sign and Gottron papules when violaceous erythematous macules and papules, respectively, are present on the interphalangeal joints and metacarpophalangeal joints. ${ }^{2}$ In addition, highly characteristic lesions include heliotrope erythema or edema of the periorbital area and visible periungual telangiectasia with dystrophic cuticles. ${ }^{2}$ Other cutaneous findings that can be seen in DM include poikiloderma atrophicans vasculare, mechanic's hands, and calcinosis cutis. ${ }^{2}$

The presence of typical cutaneous findings along with proximal muscle weakness and laboratory evidence of myositis define the classic DM. ${ }^{3}$ If patients present with the characteristic cutaneous lesions for 6 months or longer but without any clinical evidence of muscle weakness, they are 
classified as CADM. ${ }^{3}$ To further classify CADM, additional diagnostic tests such as blood work up (muscle enzymes, liver enzymes, aldolase and lactate dehydrogenase (LDH)), muscle biopsy, and electrophysiologic studies can be done. If the tests are suggestive of myositis, patients are classified as hypomyopathic DM, otherwise the patient has amyopathic DM, also called DM Sine Myositis. ${ }^{3}$

\section{CASE}

A 40-year-old Filipino male with a 10-pack year history of smoking, presented with a 5-month history of multiple pruritic well demarcated symmetric round to irregularly shaped erythematous-violaceous macules, papules, and patches on the anterior neck and chest ( $\mathrm{V}$ sign) (Figure
1A, 1D), upper back and posterior neck involvement (Shawl Sign) (Figure 1B, 1E) and scalp, face with upper eyelid involvement (Heliotrope rash), and nasolabial fold sparing (Figure 1C); also seen were multiple pruritic linear-irregularly shaped erythematous plaques on the proximal interphalangeal, metacarpophalangeal, and distal interphalangeal joints of the hands (Gottron papules) (Figure $1 \mathrm{~F}$ ) and on the left lower back (flagellate erythema) (Figure 1B). Multiple dilated tortuous blood vessels were seen on nail fold dermoscopy (Figure 2). There were no complaints of proximal muscle weakness and manual motor testing was normal. The classic lesions of dermatomyositis (DM) without a clinical evidence of myositis led to the diagnosis of clinically amyopathic dermatomyositis (CADM). There were no symptoms of a possible underlying malignancy at
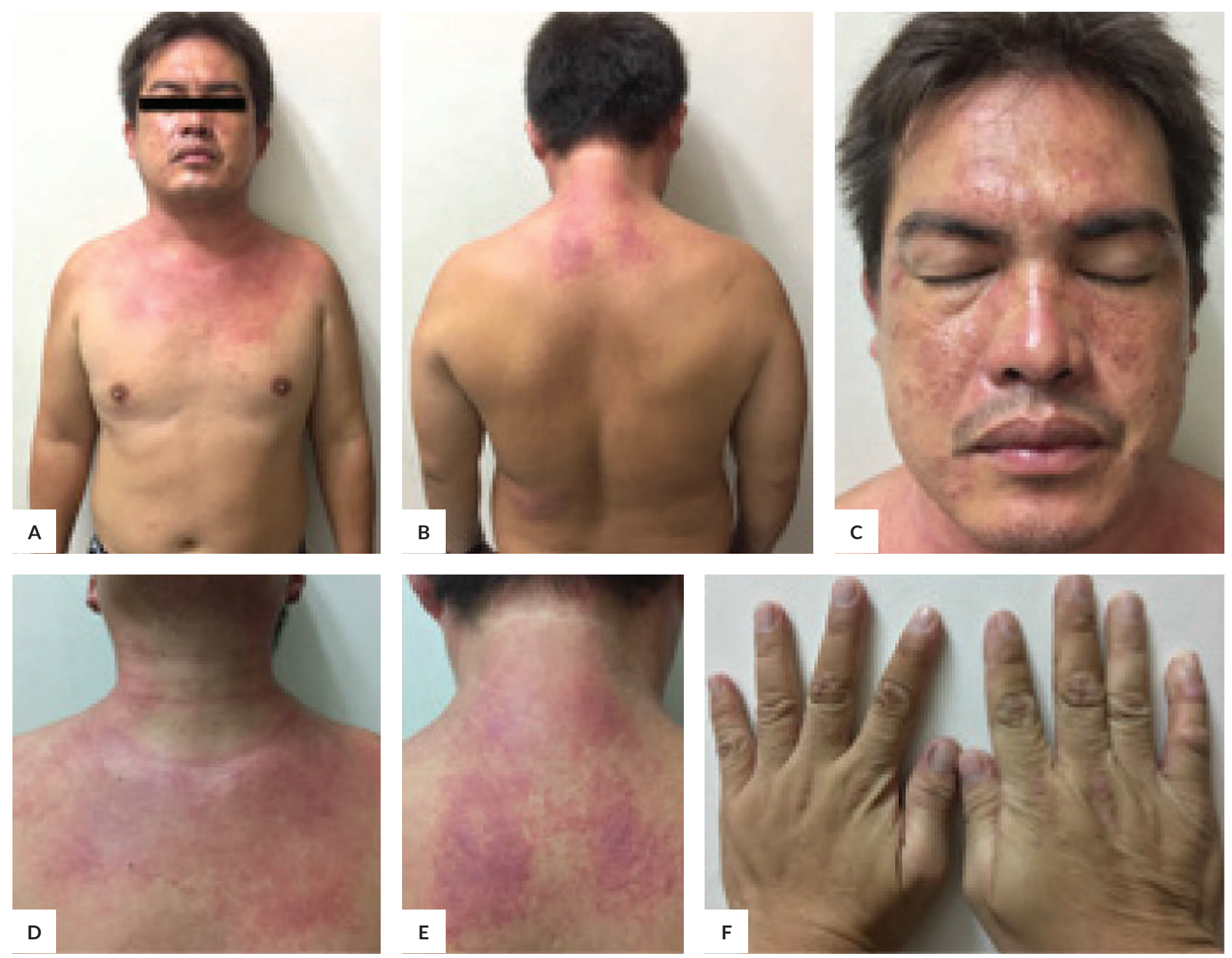

Figure 1. A. V sign: Well demarcated irregularly shaped erythematous-violaceous patches on the anterior chest. B. Shawl Sign and flagellate erythema: well-demarcated irregularly shaped erythematous-violaceous patches on the posterior neck and upper back and on the lower back. C. Note the subtle periorbital erythema and nasolabial fold sparing. D. Closer view of the $V$ sign showing submental sparing. E. Closer view of the Shawl sign with note of skin fold sparing. F. Gottron papules: multiple linear irregularly shaped plaques on the proximal interphalangeal, metacarpophalangeal and distal interphalangeal joints, more pronounced on the right hand. 


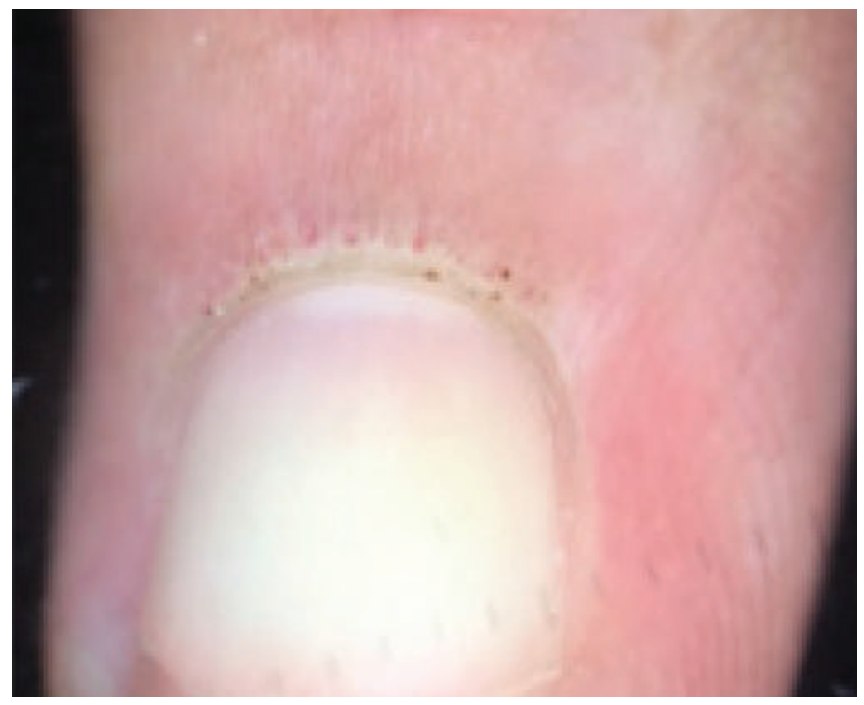

Figure 2. Nail fold telangiectasia. The dilated tortuous vessels were not grossly visible and were only appreciated with nail fold dermoscopy.
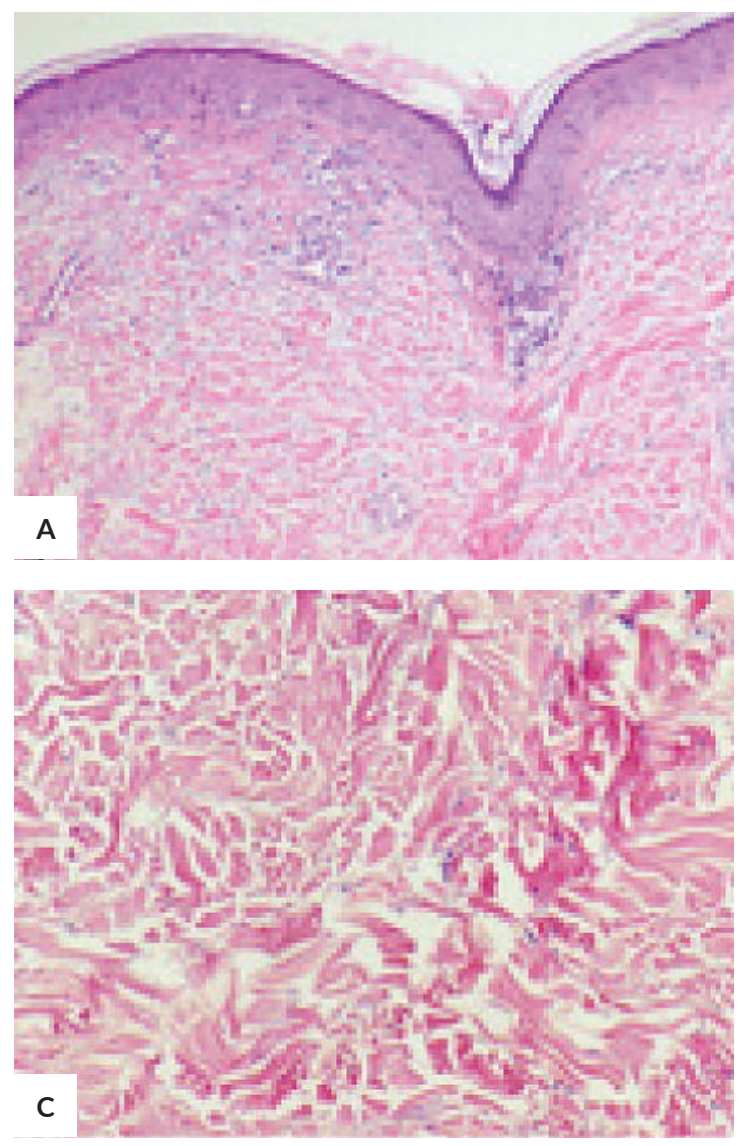

the initial consult: fever, weight loss, dysphagia, hoarseness, dyspnea, abdominal pain, and urinary and bowel changes. Systemic physical examination was also normal.

Histological examination of a plaque on the knuckle and a macule on the chest both revealed interface dermatitis with increased mucin deposition in the reticular dermis consistent with dermatomyositis (Figure 3). Muscle enzyme laboratory tests (creatine kinase-total, creatine kinase-MB) and liver enzyme tests (AST, ALT) were not suggestive of myositis (Table 1); further diagnostic studies, such as electromyography-nerve conduction studies, are needed to further classify the patient as hypomyopathic DM or amyopathic DM/DM Sine Myositis. Immunologic work up was negative for ANA-Immunofluorescence and Anti-Jo1. Tests for other antibodies associated with CADM were not locally available (anti-p155/140 or anti-TIF1 $\gamma$ for CADM with increased risk of malignancy, anti-p140 or anti-MDA5 CADM with increased risk of interstitial lung disease (ILD), and anti-Mi2 with association to the Shawl sign and cuticular overgrowth). ${ }^{2}$ Initial screening for an occult
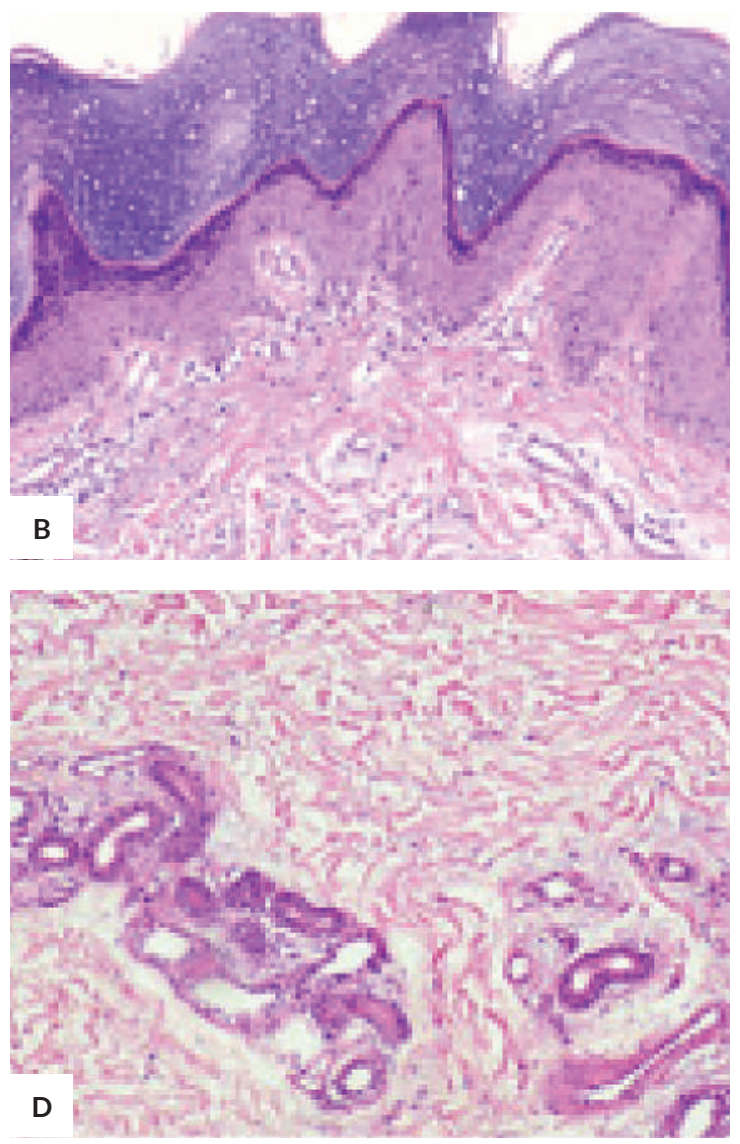

Figure 3. A. Histopathology of a patch on the chest ( -sign) (40x) showing vacuolar interface change with sparse superficial perivascular infiltrates of lymphocytes. B. Histopathology of a plaque on the metacarpophalangeal joint (Gottron papules) (40x) showing vacuolar interface change with sparse superficial perivascular infiltrate of lymphocytes with overlying compact hyperkeratosis and hypergranulosis. C. Histopathology of a patch on the chest ( $\mathrm{V}$-sign) (40x) showing mucinous deposition on the deep reticular dermis. D. Histopathology of a plaque on the metacarpophalangeal joint (Gottron papules) showing mucinous deposition on the deep reticular dermis. 
Table 1. Myositis laboratory work up done for the patient. These values were not highly suggestive of myositis hence electromyography-nerve conduction is planned for the patient

\begin{tabular}{cc}
\hline Laboratory Parameter & Value \\
CK-Total & 69 (within normal limits) \\
CK-MB & $18.9(1.18 x$ elevated $)$ \\
AST & $130(2.5 x$ elevated $)$ \\
ALT & $67(1.9 x$ elevated $)$ \\
\hline
\end{tabular}

malignancy with chest $\mathrm{X}$ ray, whole abdominal ultrasound, urinalysis, fecal occult blood test, and prostate specific antigen did not reveal any possible underlying malignancy.

Patient was then started on topical steroids (desonide $0.05 \%$ cream once a day for the face, clobetasol propionate $0.05 \%$ ointment twice a day for other affected areas), which relieved the pruritus and erythema. Sunscreen SPF 30 Broad Spectrum to be applied every 2 hours was also prescribed with decrease in erythema. However, lesions would shortly recur upon discontinuation of the topical steroids, requiring repeated courses of topical steroids. The patient did not develop signs and symptoms of muscle weakness throughout the course of consultations.

However, one month after the initial consult, the patient developed multiple cervical lymphadenopathies on level II, III and IV. Biopsy of the cervical lymphadenopathy showed metastatic undifferentiated carcinoma. With the significant history of smoking and a normal chest $\mathrm{x}$-ray, a primary nasopharyngeal cancer was initially considered. Upon consultation with the otorhinolaryngology service, a nasopharyngeal mass was detected and was subsequently biopsied. However, the histopathology revealed that the nasopharyngeal mass was a only a reactive process from the cervical lymphadenopathies showing a round cell proliferation, which was leukocyte common antigen (LCA), CD3 and CD20 positive on immunohistochemical staining. With nasopharyngeal carcinoma ruled out as the primary malignancy, the patient is still for positron emission topography (PET) scan to detect for the possible source of the metastatic undifferentiated carcinoma.

\section{DISCUSSION}

Dermatomyositis $(\mathrm{DM})$ is a rare autoimmune disease and its variant, clinically amyopathic dermatomyositis (CADM), comprise approximately 20\%-46 of DM cases., In other studies, CADM patients comprise an even lower percentage of DM at $11 \%$ to $14 \%{ }^{6,7}$ Adult patients who develop classic DM have an increased risk of having malignancy at around 20\%-24\%, ${ }^{8,9,10} \mathrm{CADM}$ has a slightly lower proportion of patients developing malignancy at around $14 \%$ to $20 \%{ }^{3,10,11}$ Hence, CADM with an associated malignancy is an unusual presentation of DM.

Since adult onset CADM patients have a definite risk of malignancy, this mandates work up to detect any underlying malignancy. Screening for a possible malignancy is done by obtaining thorough history and complete physical examination including the breast and pelvic exam in women, testicular and prostate in men, rectal examination, and nasopharyngeal examination. ${ }^{1}$ In addition, diagnostic tests that are recommended include urinalysis, stool occult blood test, serum prostate specific antigen (PSA) for men, serum CA125 mammogram and transvaginal pelvic ultrasound for women, imaging studies (CT of the chest, abdomen, and pelvis) and upper endoscopy and/or colonoscopy if with iron deficiency anemia, and occult blood in stool. ${ }^{1}$ In this case, initial screening did not point to a possible malignancy.

In addition to screening for malignancies, knowing patients with various clinical parameters with increased risk of developing malignancy can help in determining patients for closer monitoring. Risk factors for developing malignancies in DM include being male and older than 40 years old with some studies noting a higher cut-off at 45 years old. ${ }^{12,13}$ Symptoms of dysphagia, dyspnea, muscle weakness, and arthralgia are all associated with increased risk of malignancy. ${ }^{8}$ Clinical findings of cutaneous necrosis, heliotrope erythema and presence of anti-p155/140 or antiTIF1 $\gamma$ are also associated with developing malignancy. ${ }^{8}$ For this patient, being male, 40 years old, and presence of heliotrope erythema were the risk factors for malignancy. In this case, the patient developed cervical lymphadenopathies that were already metastatic undifferentiated carcinoma 1 month after the initial consult. This demonstrates that close follow up should be done especially during the first year wherein the risk of developing malignancy is highest. ${ }^{14}$

The importance of finding the primary carcinoma cannot be overemphasized as the leading cause of death in patients with DM is the metastatic spread of the malignancy rather than the complications of myositis. ${ }^{2}$ Additionally, definitive therapy of the carcinoma also results in the resolution of the DM. ${ }^{2}$ In men, the most common malignancies associated with CADM are cancers of the nasopharynx, bladder, colon and rectum, lung, and prostate. ${ }^{15}$ In Asian countries, nasopharyngeal carcinoma (NPCA) is a malignancy commonly associated with DM. 5,13,16,17 Metastatic disease of unknown primary source, such as seen in this case, was seen in 7\% of DM patients. ${ }^{15}$ Less common malignancies include cancers of the esophagus, stomach, pancreas, testes, kidney, thymus, and thyroid. ${ }^{15}$

With the 10-pack year history of smoking and NPCA being common in Asian men with CADM, NPCA was the initial consideration for the source of undifferentiated carcinoma of the patient. This led to detection of a nasopharyngeal mass by the otorhinolaryngology service. Histopathological examination of the nasopharyngeal mass only revealed a reactive process from the metastatic cervical lymphadenopathies. With the smoking history, lung cancer is also a consideration even with the normal chest $\mathrm{x}$-ray. However, less common malignancies as mentioned cannot be completely ruled out. Hence for 
this patient, positron emission tomography (PET) scan is needed to detect all the possible sources of the metastatic undifferentiated carcinoma.

In addition to carcinomas, another complication that might be seen in patients with CADM in particular is interstitial lung disease (ILD), which can be detected by anti-CADM-140 or anti-MDA5.,218 This antibody is present in up to $70 \%$ of CADM patients of which $93 \%$ had ILD. ${ }^{19,20}$ Asian patients with CADM compared to DM had a poorer survival outcome with $50 \%$ having a rapidly progressing ILD within 1 year. ${ }^{19}$ Although not locally available, this antibody can help determine the chances of ILD developing in this patient.

\section{CONCLUSION}

This case demonstrates two unique features to an already rare disease that is DM. First, this patient presented with only the characteristic cutaneous lesions of photodistributed violaceous patches with the pathognomonic Gottron papules without muscle weakness. This led to a diagnosis of CADM, which is an uncommon variant of DM. Second, this case also developed a metastatic undifferentiated carcinoma. This emphasizes the importance of malignancy screening in patients with CADM. In summary, this case highlights the role of dermatologists in detecting CADM and screening patients for development of malignancy.

\section{Statement of Authorship}

All authors approved the final version submitted.

\section{Author Disclosure}

All authors declared no conflict of interest.

\section{Funding Source}

This paper was funded by the authors. No external funding agency.

\section{REFERENCES}

1. Jorizzo JL, Vleugels RA. Dermatomyositis. In: Bolognia JL, Jorizzo JL, Schaffer J V., eds. Dermatology. 3rd ed. Elsevier Saunders; 2012:631-41.

2. Sontheimer RD, Hansen CB, Costner MI. Dermatomyositis. In: Goldsmith LA, Katz SI, Gilchrest BA, Paller AS, Leffell DJ, Wolff K, eds. Fitzpatrick's Dermatology in General Medicine. 8th editio. McGraw Hill Publishers; 2012:1926-41.

3. Gerami P1, Schope JM, McDonald L, Walling HW, Sontheimer RD. A systematic review of adult-onset clinically amyopathic dermatomyositis (dermatomyositis siné myositis): a missing link within the spectrum of the idiopathic inflammatory myopathies. J Am Acad Dermatol. 2006;54(4):597-613.
4. Bendewald MJ, Wetter DA, Li X, Davis MDP. Incidence of Dermatomyositis and Clinically Amyopathic Dermatomyositis. Arch Dermatol. 2010;146(1):26-30. doi:10.1001/archdermatol.2009.328.

5. Ang P, Sugeng M, Chua S. Classical and amyopathic dermatomyositis seen at the National Skin Centre of Singapore: a 3-year retrospective review of their clinical characteristics and association with malignancy. Ann Acad Med Singapore. 2000;29(2):219-23.

6. Ortigosa LCM, dos Reis VMS. Dermatomyositis: Analysis of 109 patients surveyed at the Hospital das Clínicas (HCFMUSP), São Paulo, Brazil. An Bras Dermatol. 2014;89(5):719-27. doi:10.1590/ abd1806-4841.20143422.

7. Chen YJ, Wu CY, Shen JL. Predicting factors of malignancy in dermatomyositis and polymyositis: A case-control study. Br J Dermatol. 2001;144(4):825-31. doi:10.1046/j.1365-2133.2001.04140.x.

8. Di Rollo D, Abeni D, Tracanna M, Capo A, Amerio P. Cancer risk in dermatomyositis: a systematic review of the literature. G Ital Dermatol Venereol. 2014;149(5):525-37. http://www.ncbi.nlm.nih. gov/pubmed/24975953.

9. Wang J, Guo G, Chen G, Wu B, Lu L, Bao L. Meta-analysis of the association of dermatomyositis and polymyositis with cancer. Br J Dermatol. 2013;169(4):838-47. doi:10.1111/bjd.12564.

10. Azuma K, Yamada H, Ohkubo M, et al. Incidence and predictive factors for malignancies in 136 Japanese patients with dermatomyositis, polymyositis and clinically amyopathic dermatomyositis. Mod Rheumatol. 2011;21(2):178-83. doi:10.1007/s10165-010-0362-y.

11. Galimberti F, Li Y, Fernandez AP. Clinically amyopathic dermatomyositis: clinical features, response to medications and malignancy-associated risk factors in a specific tertiary-care-centre cohort. Br J Dermatol. 2016;(174):158-64. doi:10.1111/bjd.14227.

12. Lu X, Yang H, Shu X, et al. Factors predicting malignancy in patients with polymyositis and dermatomyostis: A systematic review and meta-analysis. PLoS One. 2014;9(4):e94128. doi:10.1371/journal. pone.0094128.

13. Fang YF, Wu YJJ, Kuo CF, Luo SF, Yu KH. Malignancy in dermatomyositis and polymyositis: analysis of 192 patients. Clin Rheumatol. 2016;35(8):1977-84. doi:10.1007/s10067-016-3296-8.

14. Qiang JK, Kim WB, Baibergenova A, Alhusayen R. Risk of Malignancy in Dermatomyositis and Polymyositis. J Cutan Med Surg. 2017;21(2):131-6. doi:10.1177/1203475416665601.

15. Udkoff J, Cohen PR. Amyopathic Dermatomyositis : A Concise Review of Clinical Manifestations and Associated Malignancies. Am J Clin Dermatol. 2016;17(5):509-18. doi:10.1007/s40257-0160199-z.

16. Huang YL, Chen YJ, Lin MW, et al. Malignancies associated with dermatomyositis and polymyositis in Taiwan : a nationwide population-based study. 2009:854-60. doi:10.1111/j.13652133.2009.09274.x.

17. Liu WC, Ho M, Koh WP, et al. An 11-year review of dermatomyositis in asian patients. Ann Acad Med Singapore. 2010;39(11):843-7.

18. Gunawardena H, Betteridge ZE, McHugh NJ. Myositisspecific autoantibodies: Their clinical and pathogenic significance in disease expression. Rheumatology. 2009;48(6):607-12. doi:10.1093/ rheumatology/kep078.

19. Ghazi E, Sontheimer RD, Werth VP. The importance of including amyopathic dermatomyositis in the idiopathic inflammatory myositis spectrum. Clin Exp Rheumatol. 2013;31(1):128-34. doi:10.1016/j. pmrj.2014.02.014.Lumbar.

20. Hamaguchi Y, Kuwana M, Hoshino K, et al. Clinical Correlations With Dermatomyositis-Specific Autoantibodies in Adult Japanese Patients With Dermatomyositis. Arch Dermatol. 2011;147(4):391-8. 